\title{
Adaptive Synchronization for Two Different Stochastic Chaotic Systems with Unknown Parameters via a Sliding Mode Controller
}

\author{
Zengyun Wang, ${ }^{1}$ Lihong Huang, ${ }^{2,3}$ Xuxin Yang, ${ }^{1}$ and Dingyang Lu ${ }^{1}$ \\ ${ }^{1}$ Department of Mathematics, Hunan First Normal University, Changsha 410205, China \\ ${ }^{2}$ College of Mathematics and Econometrics, Hunan University, Changsha 410082, China \\ ${ }^{3}$ Hunan Women's University, Changsha 410003, China \\ Correspondence should be addressed to Zengyun Wang; shunshuang1953@163.com
}

Received 31 January 2013; Accepted 19 March 2013

Academic Editor: René Yamapi

Copyright ( 2013 Zengyun Wang et al. This is an open access article distributed under the Creative Commons Attribution License, which permits unrestricted use, distribution, and reproduction in any medium, provided the original work is properly cited.

\begin{abstract}
This paper investigates the problem of synchronization for two different stochastic chaotic systems with unknown parameters and uncertain terms. The main work of this paper consists of the following aspects. Firstly, based on the Lyapunov theory in stochastic differential equations and the theory of sliding mode control, we propose a simple sliding surface and discuss the occurrence of the sliding motion. Secondly, we design an adaptive sliding mode controller to realize the asymptotical synchronization in mean squares. Thirdly, we design an adaptive sliding mode controller to realize the almost surely synchronization. Finally, the designed adaptive sliding mode controllers are used to achieve synchronization between two pairs of different stochastic chaos systems (Lorenz-Chen and $\mathrm{Chen}-\mathrm{Lu}$ ) in the presence of the uncertainties and unknown parameters. Numerical simulations are given to demonstrate the robustness and efficiency of the proposed robust adaptive sliding mode controller.
\end{abstract}

\section{Introduction}

In the past few years, chaotic synchronization has received particular interests [1-3] mainly due to its wide applications in secure communications, ecological systems, system identification, and so forth. During the past decades, many methods and experimental techniques have been presented to realize the synchronization of two identical chaotic systems [4-10], such as adaptive control $[4,5]$, sliding mode control $[6,7]$, nonlinear feedback control $[8,9]$, and fuzzy system based control [10]. Among all these methods, sliding mode control method has been used widely to treat the unknown parameters and uncertainties [11, 12]. For example, synchronization and finite synchronization between two different chaotic systems with uncertainties and unknown parameters via sliding mode method are discussed in $[13,14]$, respectively. However, we have noted that in all of the above mentioned papers, the chaotic systems are deterministic differential equations without any random parameters or random excitation.
Recently, the stochastic modeling has played an important role in engineering application $[15,16]$ and there are some works in the field of control and synchronization on stochastic neural networks [17-25]. In accordance with the Lyapunov control theory, synchronization of stochastic delayed neural networks has been investigated in terms of linear matrix inequalities in [17]. Reference [18] discussed the adaptive lag synchronization between stochastic neural networks with time delay and [19] discussed the lag synchronization between stochastic neural networks with unknown parameters using adaptive control method. Reference [20] considered the robust decentralized adaptive control for stochastic delayed Hopfield neural networks using sling mode control method and $[21,22]$ discussed the almost surely exponential stability for stochastic neural networks. The almost surely synchronization between different stochastic chaotic systems is discussed in [23] using linear matrix equality technique. However, the parameters of the system need to be known, and the authors have not considered the chaotic system contained unknown parameters and uncertainties. 
In [24], the authors designed an adaptive controller to make sure the synchronization error trajectories between two different stochastic Chua's systems enter a small zone around zero. The control of unstable periodic orbits of stochastic chaos is discussed in [25] using sliding mode method. As far as we know, there are no results on the asymptotical synchronization and almost surely synchronization for two different stochastic chaotic systems using adaptive sliding mode control method.

In this paper, we discussed the asymptotical synchronization and almost surely synchronization for two different stochastic chaotic systems with unknown parameters and uncertain terms using sliding mode method. The structure of this paper is outlined as follows. In Section 2, we introduce the model of chaotic systems with unknown parameters and uncertain terms and give several assumptions, definitions, and lemmas. Section 3 presents the main results of this paper; we design two adaptive sliding mode controllers to realize the synchronization. Numerical examples are given in Section 4 to show the effectiveness of our proposed results. Finally, some concluding remarks are made in Section 5.

\section{Problem Statement and Mathematic Preliminaries}

In this paper, we consider the following stochastic systems with uncertain parameters in the following form:

$$
\begin{aligned}
\mathrm{d} x_{i}= & \left(f_{i}\left(x_{1}, \ldots, x_{n}\right)+F_{i}\left(x_{1}, \ldots, x_{n}\right) \theta\right. \\
& \left.+\Delta f_{i}\left(x_{1}, \ldots, x_{n}\right)\right) \mathrm{d} t \\
& +\sigma_{i}\left(x_{1}, \ldots, x_{n}\right) \mathrm{d} w(t), \quad i=1,2, \ldots, n
\end{aligned}
$$

or, in a compact form:

$$
\mathrm{d} x=(f(x)+F(x) \theta+\Delta f(x)) \mathrm{d} t+\sigma(x) \mathrm{d} w(t) .
$$

We consider the model (1) as the drive system. The response chaotic system is

$$
\begin{aligned}
\mathrm{d} y_{i}= & \left(g_{i}\left(y_{1}, \ldots, y_{n}\right)+G_{i}\left(y_{1}, \ldots, y_{n}\right) \theta\right. \\
& \left.+\Delta g_{i}\left(y_{1}, \ldots, y_{n}\right)+u_{i}(t)\right) \mathrm{d} t \\
& +\sigma_{i}\left(y_{1}, \ldots, y_{n}\right) \mathrm{d} w(t), \quad i=1,2, \ldots, n
\end{aligned}
$$

or, in a compact form:

$$
\mathrm{d} y=(g(y)+G(y) \theta+\Delta g(y)+u(t)) \mathrm{d} t+\sigma(y) \mathrm{d} w(t),
$$

where $x, y \in \mathfrak{R}^{n}$ are the state vectors, $u(t) \in \mathfrak{R}^{n}$ is the control input, $f(x), g(y) \in C^{1}\left(\Re^{n}, \mathfrak{R}^{n}\right), F(x), G(y) \in$ $C^{1}\left(\Re^{n}, \mathfrak{R}^{n \times m}\right)$, and $\sigma(x), \sigma(y) \in C^{1}\left(\mathfrak{R}^{n}, \mathfrak{R}^{n \times p}\right)$ are function matrices of $x$ and $y$, respectively. $\theta \in \mathfrak{R}^{m}$ and $\psi \in \mathfrak{R}^{m}$ are the vectors of uncertain parameters, $\Delta f(x)$ and $\Delta g(y)$ represent the nonlinear vectors that may include unknown uncertainties and other external disturbances for the master system and slave system, and $w(t)$ is a $p$-dimensional Brown motion defined on a complete probability space $(\Omega, \mathscr{F}, \mathscr{P})$ with a natural filtration $\left\{\mathscr{F}_{t}\right\}$ generated by $\{w(s): 0 \leq s \leq t\}$, where we associate $\Omega$ with the canonical space generated by $\{w(t)\}$ and denoted $\mathscr{F}$ the associated $\sigma$-algebra generated by $\{w(t)\}$ with the probability measure $\mathscr{P}$. Here the white noise $\mathrm{d} w_{i}(t)$ is independent of $\mathrm{d} w_{j}(t)$ for $i \neq j$.

(A1) The networks (2) and (4) are chaotic, and if $x, y$ are bounded, then $\left|\Delta f_{i}(x)\right|,\left|\Delta g_{i}(x)\right|$ are bounded and satisfy $\Delta f_{i}(0)=\Delta g_{i}(0)=0$ for $i=1, \ldots, n$, that is,

$$
\left|\Delta f_{i}(x)\right| \leq \alpha_{i}, \quad\left|\Delta g_{i}(x)\right| \leq \beta_{i},
$$

where $\alpha_{i}$ and $\beta_{i}$ are unknown parameters.

(A2) The noise intensity function matrices $\sigma_{i}: R^{n} \rightarrow$ $R^{p}$ are locally Lipschitz continuous and satisfying the following condition. Moreover, $\sigma_{i}$ satisfies

$$
\left[\sigma_{i}(x)-\sigma_{i}(y)\right]^{\mathrm{T}}\left[\sigma_{i}(x)-\sigma_{i}(y)\right] \leq \sum_{j=1}^{n} k_{i j}\left(x_{j}-y_{j}\right)^{2},
$$

where $k_{i j}$ are unknown parameters.

Remark 1. Note that condition (A1) is very weak. We do not impose the usual conditions such as Lipschitz condition and differentiability on the unknown uncertainties functions. It can be discontinuous or even impulsive functions. Since the trajectories of chaotic systems are always bounded, hence, condition (A1) can be easily satisfied.

Remark 2. The condition (A2) is the linear growth condition in fact, it is easy to see this condition is equivalent to the condition in [23].

Throughout this paper, we always assume the nonlinear function matrix satisfies $f(0)=g(0)=F(0)=G(0)=0$. It implies that (2) and (4) have a unique global solution on $t \geq 0$ for the initial conditions since (A1) and (A2) hold [26].

Let $e(t)=x(t)-y(t)$, then with subtracting (3) from (1) the error dynamics is obtained as follows:

$$
\begin{aligned}
\mathrm{d} e_{i}=( & f_{i}(x)-g_{i}(y)+F_{i}(x) \theta-G(y) \psi+\Delta f_{i}(x) \\
& \left.-\Delta g_{i}(y)-u_{i}(t)\right) \mathrm{d} t+\left(\sigma_{i}(x)-\sigma_{i}(y)\right) \mathrm{d} w(t) .
\end{aligned}
$$

It is clear that the synchronization problem can be transformed to be the equivalent problem of stabilizing the error system (7).

Remark 3. From the mathematical point of view, the model is more general. If the noise intensity function matrices $\sigma(x)=$ $\sigma(y)=0$, (7) becomes the model in [13]. Furthermore, if $\Delta f(x)=\Delta g(y)=0$, the system will be the model in [14]. If $\Delta f(x)=\Delta g(y)=0$ and $\theta, \psi$ are the known vectors, this will be the model discussed in [23].

Definition 4 (see [19]). The error system (7) is said to be globally stable in mean squares if for any given initial condition such that

$$
\lim _{t \rightarrow \infty} E\|e(t)\|^{2}=0
$$

where $E[\cdot]$ is the mathematical expectation. 
Definition 5 (see [26]). The trivial solution of the error system (7) is said to be almost surely exponentially stable, if for almost all sample paths of the solution $e(t)$, we have

$$
\limsup _{t \rightarrow \infty} \frac{1}{t} \log \|e(t)\|<0,
$$

that is, the the drive system and response system are almost surely synchronization.

Remark 6. If $e(t)=0$, this means that $x(t)=y(t)$, so $u(t)=f(x)+F(x) \theta+\Delta f(x)-g(y)-G(y) \psi-\Delta g(x)+$ $(\sigma(x)-\sigma(y)) \dot{w}(t)$, where $\dot{w}(t)=\mathrm{d} w(t) / \mathrm{d} t$. This implies that $u(t)$ directly depends on white Gaussian noises and it is an accessible causal signal; this means that the synchronization cannot be realized completely.

The purpose of this paper is to consider the adaptive feedback synchronization problem for stochastic chaotic systems with unknown parameters and uncertainties. The main work of this paper consists of the following aspects. (i) Design an adaptive controller such that the asymptotical stability of the error system (7) can be achieved in mean squares that $\lim _{t \rightarrow \infty} E\|e(t)\|^{2}=0$. (ii) Design an adaptive control such that the error system (7) can be almost surely stable, that means the almost surely synchronization could be achieved between drive system and response system.

Before proposing the main results, we introduce some lemmas which will be used in the following sections.

Lemma 7 (see [20, 27]). The trivial solution of a stochastic differential equation as follows

$$
\mathrm{d} x(t)=a(t, x) \mathrm{d} t+b(t, x) \mathrm{d} \omega(t),
$$

with $a(t, x)$ and $b(t, x)$ sufficiently differentiable maps, is globally asymptotically stable in probability if there exists a function $V(t, x)$ which is positive definite in the Lyapunov sense and satisfies

$$
\begin{aligned}
\mathscr{L} V(t, x)= & V_{t}(t, x)+V_{x}(t, x) \cdot a(t, x) \\
& +\frac{1}{2} \operatorname{tr}\left\{b^{\mathrm{T}}(t, x) V_{x x}(t, x) b(t, x)\right\}<0,
\end{aligned}
$$

for $x \neq 0$, where $V_{t}(t, x)=\partial V(t, x) / \partial t, V_{x}(t, x)=\partial V(t, x) / \partial x$, and $V_{x x}(t, x)=\left(\partial^{2} V(t, x) / \partial x_{i} \partial x_{j}\right)_{n \times n}$.

Lemma 8 (see $[23,26])$. Suppose there exist a nonnegative function $V(x, t) \in C^{2,1}\left(R^{n} \times\left[t_{0},+\infty\right), R^{+}\right)$and three positive numbers $p, \alpha$, and $\lambda$ such that, for all $x \neq 0$ and $t \geq t_{0}$,

$$
\alpha|x|^{p} \leq V(x, t), \quad \mathscr{L} V(t, x)<-\lambda V(t, x)
$$

holds, then for any $x_{0} \in R^{n}$, the trivial solution of (10) is almost surely exponentially asymptotically stable; that is,

$$
\limsup _{t \rightarrow \infty} \frac{1}{t} \log \left|x\left(t, t_{0}, x_{0}\right)\right|<-\frac{\lambda}{p}
$$

holds almost surely.

\section{Main Results}

To design the adaptive feedback controller to realize the synchronization for stochastic chaotic systems with unknown parameters and uncertainties, we use the sliding mode control method. In this section, the nonsingular terminal sliding mode is chosen as

$$
s_{i}(t)=\lambda_{i} e_{i}(t), \quad i=1, \ldots, n,
$$

where $s_{i}(t) \in R, s(t)=\left[s_{1}(t), s_{2}(t), \ldots, s_{n}(t)\right]^{\mathrm{T}}$ and $\lambda_{i}>0$ are constants.

3.1. Design of an Adaptive Controller to Realize Asymptotical Synchronization in Mean Squares. In this section, we are going to design an adaptive controller with updating laws such that the state trajectories will move to the sliding surface in mean squares. To ensure the occurrence of the sliding motion, an adaptive sliding mode controller is proposed as

$$
\begin{aligned}
u_{i}(t)= & f_{i}(x)+F_{i}(x) \widehat{\theta}-g_{i}(y)-G_{i}(y) \widehat{\psi} \\
& +\left(\widehat{\alpha}_{i}+\widehat{\beta}_{i}\right) \operatorname{sign}\left(s_{i}(t)\right)+k_{i} s_{i}(t) \\
& +\sum_{j=1}^{n} \frac{\lambda_{j}^{2}}{\lambda_{i}^{3}} \widehat{k}_{j i} s_{i}(t), \quad i=1,2, \ldots, n,
\end{aligned}
$$

where $\widehat{\theta}, \widehat{\psi}, \widehat{\alpha}_{i}, \widehat{\beta}_{i}, \widehat{k}_{i j}$ are the estimations for $\theta, \psi, \alpha_{i}, \beta_{i}, k_{i j}$, respectively. $k_{i}>0$ is the switching gain and a constant, $i=$ $1,2, \ldots, n$.

To tackle the uncertainties and unknown parameters, appropriate adaptive laws are defined as follows:

$$
\begin{array}{lc}
\dot{\hat{\theta}}=F^{\mathrm{T}}(x) \gamma(t), & \widehat{\theta}(0)=\widehat{\theta}_{0} \\
\dot{\hat{\psi}}=-G^{\mathrm{T}}(y) \gamma(t), & \widehat{\psi}(0)=\widehat{\psi}_{0} \\
\dot{\hat{\alpha}}_{i}=\lambda_{i}\left|s_{i}(t)\right|, & \widehat{\alpha}_{i}(0)=\widehat{\alpha}_{i 0} \\
\dot{\hat{\beta}}_{i}=\lambda_{i}\left|s_{i}(t)\right|, & \widehat{\beta}_{i}(0)=\widehat{\beta}_{i 0} \\
\dot{\hat{k}}_{i j}=\frac{\lambda_{i}^{2}}{\lambda_{j}^{2}} s_{j}^{2}(t), & \widehat{k}_{i j}(0)=\widehat{k}_{i j 0}
\end{array}
$$

where $\gamma(t)=\left[\lambda_{1} s_{1}(t), \lambda_{2} s_{2}(t), \ldots, \lambda_{n} s_{n}(t)\right]^{\mathrm{T}}$, and $\widehat{\theta}_{0}, \widehat{\psi}_{0}$, $\widehat{\alpha}_{i 0}$, and $\widehat{\beta}_{i 0}$ are the initial values of the update parameters, respectively.

The proposed control input in (15) with the updating laws in (16) will guarantee the reaching condition $\lim _{t \rightarrow \infty} E\|s(t)\|^{2}=0$ and ensure the occurrence of the sliding motion, which is proved in the following theorem.

Theorem 9. Suppose that the assumption conditions (A1) and (A2) hold; consider the error dynamics (7); this system is controlled by $u(t)$ in (15) with updating laws in (16), then the error system trajectories will converge to the sliding surface $s(t)=0$ in mean squares. 
Proof. Select a positive definite function as a Lyapunov function candidate in the form of

$$
\begin{aligned}
V(t)= & \frac{1}{2} \sum_{i=1}^{n}\left(s_{i}^{2}(t)+\left(\widehat{\alpha}_{i}-\alpha_{i}\right)^{2}+\left(\widehat{\beta}_{i}-\beta_{i}\right)^{2}\right) \\
& +\frac{1}{2} \sum_{i=1}^{n} \sum_{j=1}^{n}\left(\widehat{k}_{i j}-k_{i j}\right)^{2}+\frac{1}{2}\|\hat{\theta}-\theta\|^{2}+\frac{1}{2}\|\widehat{\psi}-\psi\|^{2} .
\end{aligned}
$$

Since $\mathrm{d} s_{i}(t)=\lambda_{i} \mathrm{~d} e_{i}(t)$, by Itô's differential rule, the stochastic derivative of $V(t)$ along trajectories of error system (7) can be obtained as follows:

$$
\mathrm{d} V(t)=\mathscr{L} V(t) \mathrm{d} t+\sum_{i=1}^{n} \lambda_{i} s_{i}(t)\left(\sigma_{i}(x)-\sigma_{i}(y)\right) \mathrm{d} w(t),
$$

where the weak infinitesimal operator $\mathscr{L}$ is given by

$$
\begin{aligned}
& \mathscr{L} V(t) \\
& =\sum_{i=1}^{n}\left[\lambda _ { i } s _ { i } ( t ) \left(\left(f_{i}(x)-g_{i}(y)+F_{i}(x) \theta\right.\right.\right. \\
& \left.\left.\left.-G_{i}(y) \psi+\Delta f_{i}(x)-\Delta g_{i}(y)-u_{i}(t)\right)\right)\right] \\
& +\sum_{j=1}^{n} \lambda_{i}^{2}\left(\sigma_{i}(x(t))-\sigma_{i}(y(t))\right)^{\mathrm{T}} \\
& \times\left(\sigma_{i}(x(t))-\sigma_{i}(y(t))\right)+\left(\widehat{\alpha}_{i}-\alpha_{i}\right) \dot{\widehat{\alpha}}_{i} \\
& +\left(\widehat{\beta}_{i}-\beta_{i}\right) \dot{\hat{\beta}}_{i} \\
& +\sum_{i=1}^{n} \sum_{j=1}^{n}\left(\widehat{k}_{i j}-k_{i j}\right) \dot{\widehat{k}}_{i j}+(\widehat{\theta}-\theta)^{\mathrm{T}} \dot{\hat{\theta}}+(\widehat{\psi}-\psi)^{\mathrm{T}} \dot{\hat{\psi}} \\
& \leq \sum_{i=1}^{n}\left[\lambda_{i} s_{i}(t) F_{i}(x)(\theta-\widehat{\theta})-\lambda_{i} s_{i}(t) G_{i}(y)(\psi-\widehat{\psi})\right. \\
& +\alpha_{i} \lambda_{i}\left|s_{i}(t)\right|+\beta_{i} \lambda_{i}\left|s_{i}(t)\right|-\widehat{\alpha}_{i} \lambda_{i}\left|s_{i}(t)\right| \\
& -\widehat{\beta}_{i} \lambda_{i}\left|s_{i}(t)\right|-k_{i} \lambda_{i} s_{i}(t)^{2} \\
& -\lambda_{i} s_{i}(t) \sum_{j=1}^{n} \frac{\lambda_{j}^{2}}{\lambda_{i}^{3}} \widehat{k}_{j i} s_{i}(t)+\sum_{j=1}^{n} \lambda_{i}^{2} k_{i j} e_{j}^{2}(t) \\
& \left.+\left(\widehat{\alpha}_{i}-\alpha_{i}\right) \dot{\hat{\alpha}}_{i}+\left(\widehat{\beta}_{i}-\beta_{i}\right) \dot{\hat{\beta}}_{i}\right] \\
& +\sum_{i=1}^{n} \sum_{j=1}^{n}\left(\widehat{k}_{i j}-k_{i j}\right) \dot{\hat{k}}_{i j}+(\widehat{\theta}-\theta)^{\mathrm{T}} \dot{\hat{\theta}}+(\widehat{\psi}-\psi)^{\mathrm{T}} \dot{\hat{\psi}}
\end{aligned}
$$

Using the facts $\sum_{i=1}^{n} \lambda_{i} s_{i}(t) F_{i}(x) \theta=\theta^{\mathrm{T}} F^{\mathrm{T}}(x) \gamma(t)$, $\sum_{i=1}^{n} \lambda_{i} s_{i}(t) G_{i}(x) \psi=\psi^{\mathrm{T}} G^{\mathrm{T}}(x) \gamma(t)$ and the updating laws in (16), one has

$$
\mathscr{L} V(t) \leq-\sum_{i=1}^{n} k_{i} \lambda_{i} s_{i}^{2}=-\sum_{i=1}^{n} \eta_{i} s_{i}^{2}=-\eta s^{\mathrm{T}}(t) s(t),
$$

where $\eta_{i}=k_{i} \lambda_{i}, i=1,2, \ldots, n$ and $\eta=\min \left\{\eta_{1}, \eta_{2}, \ldots, \eta_{n}\right\}>$ 0 .

Taking mathematical expectation on both sides of (20), in view of (18) and the definition of $V(t)$, we obtain

$$
\frac{1}{2} E\|s(t)\|^{2} \leq E V(t) \leq E V(0)-\eta \int_{0}^{t} E\|s(\tau)\|^{2} \mathrm{~d} \tau .
$$

Based on the LaSalle invariance principle of stochastic differential equation, which was developed in $[28,29]$, we have $s(t) \rightarrow 0$ when $t \rightarrow \infty$, which in turn illustrates that $\lim _{t \rightarrow \infty} E\|s(t)\|^{2}=0$. This complete the proof.

Remark 10. If $\sigma(x)=\sigma(y)=0$, this theorem is an extension of Theorem 1 in [13]. If $\sigma(x)=\sigma(y)=0$ and $\Delta f(x)=\Delta g(x)$, this is a similar result of Theorem 2 in [14].

Remark 11. Since the control law (15) contains the sign function as a hard switcher, the undesirable chattering phenomenon occurs. According to Lemma 2 and Remark 2 in [13], we can replace the $\operatorname{sign}\left(s_{i}\right)$ function by $\tanh \left(\epsilon s_{i}\right), \epsilon>0$.

Remark 12. From the proof of Theorem 9, we know that $\mathscr{L} V(t) \leq 0$ as long as $s(t) \neq 0$. Therefore, the trajectories $s(t)$ will converge to $s(t)=0$ in mean squares. On the other hand, from the adaptive law (18) we can see $\dot{\hat{\theta}}, \dot{\widehat{\psi}}, \dot{\widehat{\alpha}}_{i}, \dot{\widehat{\beta}}_{i}$, and $\widehat{k}_{i j}$ turn to zero when $s(t)=0$, which implies that $\widehat{\theta}, \widehat{\psi}, \widehat{\alpha}_{i}, \widehat{\beta}_{i}$, and $\widehat{k}_{i j}$ approach some constants as $s(t) \rightarrow 0$. However, this does not elaborate that $\hat{\theta} \rightarrow \theta, \widehat{\psi} \rightarrow \psi$. This point is consistent with the results of [30].

In fact, the unknown parameters $\theta, \psi$ in (7) cannot identify with $\widehat{\theta}, \widehat{\psi}$. We offer the following theorem.

Theorem 13. In Theorem 9, if $\Delta f(x) \neq \Delta g(y)$ and the synchronization between (1) and (3) is realized, the unknown parameters $\theta, \psi$ in (7) cannot identify with $\widehat{\theta}, \widehat{\psi}$ in (17), respectively.

Proof. We prove it by its contrapositive proposition. On the synchronization manifold $x(t)=y(t)$, it follows $s_{i}(t)=0$ and $\mathrm{d} s_{i}=0$. From (7) and (15), we have

$$
\begin{gathered}
0=\left[\lambda _ { i } \left(F_{i}(x)(\theta-\widehat{\theta})-G_{i}(y)(\psi-\widehat{\psi})+\Delta f_{i}(x)\right.\right. \\
\left.\quad-\Delta g_{i}(y)-\left(\widehat{\alpha}_{i}+\widehat{\beta}_{i}\right) \operatorname{sign} s_{i}(t)-k_{i} s_{i}(t)\right) \\
\left.\quad-\sum_{j=1}^{n} \frac{\lambda_{j}^{2}}{\lambda_{i}^{2}} \widehat{k}_{j i} s_{i}(t)\right] \mathrm{d} t+\lambda_{i}\left(\sigma_{i}(x(t))-\sigma_{i}(y(t))\right) \mathrm{d} w(t) .
\end{gathered}
$$

Suppose $\widehat{\theta}=\theta$ and $\widehat{\psi}=\psi$; we have $\Delta f_{i}(x)=\Delta g_{i}(y)=\Delta g_{i}(x)$; this means $\Delta f(x)=\Delta g(x)$, which is a contradiction. This completes the proof.

If $\Delta f(x)=\Delta g(y)=0$, we can get the following theorem. 
Theorem 14. Suppose that the assumption condition (A2) holds. Then under the controller (15) with updating laws (16), the response system and the drive system are asymptotical synchronized in mean squares. Moreover, if $F_{i j}(x), G_{i j}(y)$ are linearly independent of the synchronization manifold, then $\lim _{t \rightarrow \infty}(\widehat{\theta}-\theta)=\lim _{t \rightarrow \infty}(\widehat{\psi}-\psi)=0$.

Proof. It is easy to get the following error system:

$$
\begin{aligned}
\mathrm{d} e_{i}= & \left(f_{i}(x)-g_{i}(y)+F_{i}(x) \theta-G(y) \psi-u_{i}(t)\right) \mathrm{d} t \\
& +\left(\sigma_{i}(x)-\sigma_{i}(y)\right) \mathrm{d} w(t) .
\end{aligned}
$$

Define the following Lyapunov function candidate

$$
\begin{aligned}
V_{1}(t)= & \frac{1}{2} \sum_{i=1}^{n} s_{i}(t)^{2}+\frac{1}{2} \sum_{i=1}^{n} \sum_{j=1}^{n}\left(\widehat{k}_{i j}-k_{i j}\right)^{2} \\
& +\frac{1}{2}\|\hat{\theta}-\theta\|^{2}+\frac{1}{2}\|\widehat{\psi}-\psi\|^{2} .
\end{aligned}
$$

Since $\mathrm{d} s_{i}(t)=\lambda_{i} \mathrm{~d} e_{i}(t)$, by Itô's differential rule, the stochastic derivative of $V(t)$ along trajectories of error system (7) can be obtained as follows:

$$
\mathrm{d} V_{1}(t)=\mathscr{L} V_{1}(t) \mathrm{d} t+\sum_{1}^{n} \lambda_{i} s_{i}\left(\sigma_{i}(x)-\sigma_{i}(y)\right) \mathrm{d} w(t),
$$

where the weak infinitesimal operator $\mathscr{L}$ is given by

$$
\begin{aligned}
& \mathscr{L} V_{1}(t) \\
& =\sum_{i=1}^{n}\left[\lambda _ { i } s _ { i } ( t ) \left(\left(f_{i}(x)-g_{i}(y)\right.\right.\right. \\
& \left.\left.\left.+F_{i}(x) \theta-G_{i}(y) \psi-u_{i}(t)\right)\right)\right] \\
& +\sum_{j=1}^{n} \lambda_{i}^{2}\left(\sigma_{i}(x(t))-\sigma_{i}(y(t))\right)^{\mathrm{T}} \\
& \times\left(\sigma_{i}(x(t))-\sigma_{i}(y(t))\right) \\
& +\sum_{i=1}^{n} \sum_{j=1}^{n}\left(\widehat{k}_{i j}-k_{i j}\right) \dot{\hat{k}}_{i j}+(\widehat{\theta}-\theta)^{\mathrm{T}} \dot{\hat{\theta}}+(\widehat{\psi}-\psi)^{\mathrm{T}} \dot{\hat{\psi}} \\
& \leq \sum_{i=1}^{n}\left[\lambda_{i} s_{i}(t) F_{i}(x)(\theta-\widehat{\theta})\right. \\
& -\lambda_{i} s_{i}(t) G_{i}(y)(\psi-\widehat{\psi}) \\
& +\alpha_{i} \lambda_{i}\left|s_{i}(t)\right|+\beta_{i} \lambda_{i}\left|s_{i}(t)\right| \\
& -\widehat{\alpha}_{i} \lambda_{i}\left|s_{i}(t)\right|-\widehat{\beta}_{i} \lambda_{i}\left|s_{i}(t)\right|-k_{i} \lambda_{i} s_{i}(t)^{2} \\
& -\lambda_{i} s_{i}(t) \sum_{j=1}^{n} \frac{\lambda_{j}^{2}}{\lambda_{i}^{3}} \widehat{k}_{j i} s_{i}(t) \\
& \left.+\sum_{j=1}^{n} \lambda_{i}^{2} k_{i j} e_{j}(t)^{2}\right]
\end{aligned}
$$

$$
\begin{aligned}
& +\sum_{i=1}^{n} \sum_{j=1}^{n}\left(\widehat{k}_{i j}-k_{i j}\right) \dot{\hat{k}}_{i j} \\
& +(\widehat{\theta}-\theta)^{\mathrm{T}} \dot{\hat{\theta}}+(\widehat{\psi}-\psi)^{\mathrm{T}} \dot{\hat{\psi}} .
\end{aligned}
$$

From the update laws (16), we can always choose the appropriate initial values of $\widehat{\alpha}_{i 0}$ and $\widehat{\beta}_{i 0}$ to make $\widehat{\alpha}_{i}>0$ and $\widehat{\beta}_{i}>0$. Since $\widehat{\alpha}_{i} s_{i}(t) \operatorname{sign}\left(s_{i}(t)\right) \geq 0$ and $\widehat{\beta}_{i} s_{i}(t) \operatorname{sign}\left(s_{i}(t)\right) \geq$ 0 . Using the facts $\sum_{i=1}^{n} \lambda_{i} s_{i}(t) F_{i}(x) \theta=\theta^{\mathrm{T}} F^{\mathrm{T}}(x) \gamma(t)$, $\sum_{i=1}^{n} \lambda_{i} s_{i}(t) G_{i}(x) \psi=\psi^{\mathrm{T}} G^{\mathrm{T}}(x) \gamma(t)$ and the updating laws in (18), with the same procedure of the proof of Theorem 9, we also arrive at $E\|s(t)\|^{2} \rightarrow 0$.

On the synchronization manifold $x(t)=y(t)$, it follows $s(t)=0$ and $\mathrm{d} s_{i}=0$. From (7) and (15), we have

$$
0=\lambda_{i}\left[F_{i}(x)(\theta-\widehat{\theta})-G_{i}(y)(\psi-\widehat{\psi})\right] .
$$

Since $F_{i j}(x), G_{i j}(y)$ are linearly independent on the synchronization manifold, therefore, the above equality holds if and only if $\widehat{\theta}=\theta$ and $\widehat{\psi}=\psi$.

Remark 15. Certainly, under the controller without $\left(\widehat{\alpha}_{i}+\right.$ $\left.\widehat{\beta}_{i}\right)$ sign $s_{i}(t)$ term, the response system can also synchronize the drive system in mean squares. However, under the controller (15) with this term, it is more effective to realize the synchronization with this term. If $x_{i}(t)>y_{i}(t)$, this term will help to increase $y_{i}(t)$, and if $x_{i}(t)<y_{i}(t)$, this term will help to decrease $y_{i}(t)$. Hence, this term $\left(\widehat{\alpha}_{i}+\widehat{\beta}_{i}\right)$ sign $s_{i}(t)$ can enhance the synchronization speed.

3.2. Design of an Adaptive Controller to Realize Almost Surely Synchronization. In this section, we are going to design an adaptive controller with update laws such that the state trajectories will move to the sliding surface almost surely. We first introduce the following assumptions for the unknown parameters.

(A3) The unknown parameters vectors $\theta$ and $\psi$ are norm bounded with known bounds, that is,

$$
\|\theta\| \leq \bar{\theta}, \quad\|\psi\| \leq \bar{\psi},
$$

where $\bar{\theta}$ and $\bar{\psi}$ are two known positive constants.

(A4) Assume (A1) and (A2) hold, and $\alpha_{i}, \beta_{i}$, and $k_{i j}$ are known positive constants.

To ensure the occurrence of the sliding motion, an adaptive sliding mode controller is proposed as

$$
\begin{aligned}
u_{i}(t)= & f_{i}(x)+F_{i}(x) \widehat{\theta}-g_{i}(y)-G_{i}(y) \widehat{\psi} \\
+ & \left(\alpha_{i}+\beta_{i}\right) \operatorname{sign}\left(s_{i}(t)\right)+\frac{1}{2} k_{i} s_{i}(t) \\
- & \eta\left(\|\widehat{\theta}\|^{2}+\bar{\theta}^{2}+\|\widehat{\psi}\|^{2}+\bar{\psi}^{2}\right) \\
& \times\left(\frac{s_{i}(t)}{\lambda_{i}\|s(t)\|^{2}}\right)+\sum_{j=1}^{n} \frac{\lambda_{j}^{2}}{\lambda_{i}^{3}} k_{j i} s_{i}(t),
\end{aligned}
$$


where $\widehat{\theta}, \widehat{\psi}$ are the estimations for $\theta, \psi$, respectively. $\eta_{i}=$ $k_{i} \lambda_{i}, i=1,2, \ldots, n$ and $\eta=\min \left\{\eta_{1}, \eta_{2}, \ldots, \eta_{n}\right\}>0, k_{i}>0$ are the switching gain and constants, $i=1,2, \ldots, n$.

To tackle the uncertainties and unknown parameters, appropriate adaptive laws are defined as follows:

$$
\begin{aligned}
& \dot{\hat{\theta}}=F^{\mathrm{T}}(x) \gamma(t), \quad \widehat{\theta}(0)=\widehat{\theta}_{0} \\
& \dot{\hat{\psi}}=-G^{\mathrm{T}}(y) \gamma(t), \quad \widehat{\psi}(0)=\widehat{\psi}_{0},
\end{aligned}
$$

where $\gamma(t)=\left[\lambda_{1} s_{1}(t), \lambda_{2} s_{2}(t), \ldots, \lambda_{n} s_{n}(t)\right]^{\mathrm{T}}$, and $\widehat{\theta}_{0}, \widehat{\psi}_{0}$ are the initial values of the update parameters, respectively.

Based on the control input in (29) with the updating laws in (30) to guarantee the reaching condition $\lim _{t \rightarrow \infty} s(t)=$ 0 almost surely holds and to ensure the occurrence of the sliding motion, a theorem is proposed and proved.

Theorem 16. Suppose that the assumption conditions (A3) and (A4) hold; consider the error dynamics (7); this system is controlled by $u(t)$ in (29) with updating laws in (30), then the error system trajectories will converge to the sliding surface $s(t)=0$ almost surely.

Proof. Select a positive definite function as a Lyapunov function candidate in the form of

$$
V(t)=\frac{1}{2} \sum_{i=1}^{n} s_{i}(t)^{2}+\frac{1}{2}\|\hat{\theta}-\theta\|^{2}+\frac{1}{2}\|\widehat{\psi}-\psi\|^{2} .
$$

Since $\mathrm{d} s_{i}(t)=\lambda_{i} \mathrm{~d} e_{i}(t)$, by Itô's differential rule, the stochastic derivative of $V(t)$ along trajectories of error system (7) can be obtained as follows:

$$
\mathrm{d} V(t)=\mathscr{L} V(t) \mathrm{d} t+\sum_{1}^{n} \lambda_{i} s_{i}(t)\left(\sigma_{i}(x)-\sigma_{i}(y)\right) \mathrm{d} w(t),
$$

where the weak infinitesimal operator $\mathscr{L}$ is given by

$$
\begin{aligned}
& \mathscr{L} V(t) \\
& =\sum_{i=1}^{n}\left[\lambda _ { i } s _ { i } ( t ) \left(\left(f_{i}(x)-g_{i}(y)+F_{i}(x) \theta\right.\right.\right. \\
& \left.\left.\left.-G_{i}(y) \psi+\Delta f_{i}(x)-\Delta g_{i}(y)-u_{i}(t)\right)\right)\right] \\
& +\sum_{j=1}^{n} \lambda_{i}^{2}\left(\sigma_{i}(x(t))-\sigma_{i}(y(t))\right)^{\mathrm{T}}\left(\sigma_{i}(x(t))-\sigma_{i}(y(t))\right) \\
& +(\widehat{\theta}-\theta)^{\mathrm{T}} \dot{\hat{\theta}}+(\widehat{\psi}-\psi)^{\mathrm{T}} \dot{\hat{\psi}} \\
& \leq \sum_{i=1}^{n}\left[\lambda_{i} s_{i}(t) F_{i}(x)(\theta-\hat{\theta})-\lambda_{i} s_{i}(t) G_{i}(y)(\psi-\widehat{\psi})\right. \\
& -\frac{1}{2} k_{i} \lambda_{i} s_{i}(t)^{2}-\lambda_{i} s_{i}(t) \sum_{j=1}^{n} \frac{\lambda_{j}^{2}}{\lambda_{i}^{3}} k_{j i} s_{i}(t) \\
& +\lambda_{i} s_{i}(t) \eta\left(\|\widehat{\theta}\|^{2}+\bar{\theta}^{2}+\|\widehat{\psi}\|^{2}+\bar{\psi}^{2}\right)
\end{aligned}
$$

$$
\begin{gathered}
\left.\times\left(\frac{s_{i}(t)}{\lambda_{i}\|s(t)\|^{2}}\right)+\sum_{j=1}^{n} \lambda_{i}^{2} k_{i j} e_{j}^{2}(t)\right] \\
+(\widehat{\theta}-\theta)^{\mathrm{T}} \dot{\hat{\theta}}+(\widehat{\psi}-\psi)^{\mathrm{T}} \dot{\hat{\psi}} .
\end{gathered}
$$

Using the facts $\sum_{i=1}^{n} \lambda_{i} s_{i}(t) F_{i}(x) \theta=\theta^{\mathrm{T}} F^{\mathrm{T}}(x) \gamma(t)$, $\sum_{i=1}^{n} \lambda_{i} s_{i}(t) G_{i}(x) \psi=\psi^{\mathrm{T}} G^{\mathrm{T}}(x) \gamma(t)$ and the updating laws in (30), one has

$$
\begin{aligned}
& \mathscr{L} V(t) \\
& \leq-\sum_{i=1}^{n} \frac{1}{2} k_{i} \lambda_{i} s_{i}^{2}+\eta\left(\|\hat{\theta}\|^{2}+\bar{\theta}^{2}+\|\widehat{\psi}\|^{2}+\bar{\psi}^{2}\right) \\
& \leq-\eta\left[\frac{1}{2} s^{\mathrm{T}}(t) s(t)-\left(\|\hat{\theta}\|^{2}+\bar{\theta}^{2}\right)-\left(\|\widehat{\psi}\|^{2}+\bar{\psi}^{2}\right)\right] .
\end{aligned}
$$

Since $\|\widehat{\theta}-\theta\|^{2} \leq 2\left(\|\widehat{\theta}\|^{2}+\|\theta\|^{2}\right) \leq 2\left(\|\widehat{\theta}\|^{2}+\bar{\theta}^{2}\right)$ and similarly we have $\|\widehat{\psi}-\psi\|^{2} \leq 2\left(\|\widehat{\psi}\|^{2}+\bar{\psi}^{2}\right)$, so we can conclude that $-\left(\|\hat{\theta}\|^{2}+\bar{\theta}^{2}\right) \leq-1 / 2\|\widehat{\theta}-\theta\|^{2}$ and $-\left(\|\widehat{\theta}\|^{2}+\bar{\theta}^{2}\right) \leq-1 / 2\|\hat{\theta}-\theta\|^{2}$. So it is easy to get

$$
\begin{array}{r}
\mathscr{L} V(t) \leq-\eta\left[\frac{1}{2} s^{\mathrm{T}}(t) s(t)+\frac{1}{2}\|\hat{\theta}-\theta\|^{2}\right. \\
\left.+\frac{1}{2}\|\hat{\theta}-\theta\|^{2}\right] \leq-\eta V(t) .
\end{array}
$$

Then from Lemma 8, we can obtain: $\lim _{t \rightarrow \infty} s(t)=0$ almost surely. This completes the proof.

Remark 17. From the proof, it is easy to see that the positive numbers in (A4) also could be unknown; we just modify the estimate parameters in the controller. To simplify, we discussed the problem under condition (A4).

Remark 18. The controller in (30) contains a discontinuous term $\eta\left(\|\widehat{\theta}\|^{2}+\bar{\theta}^{2}+\|\widehat{\psi}\|^{2}+\bar{\psi}^{2}\right)\left(s_{i} / \lambda_{i}\|s\|^{2}\right)$ and thus chattering is unavoidable. In order to eliminate this chattering, this control term can be modified as $\eta\left(\|\widehat{\theta}\|^{2}+\bar{\theta}^{2}+\|\widehat{\psi}\|^{2}+\bar{\psi}^{2}\right)\left(s_{i} /\left(\lambda_{i}\|s\|^{2}+\epsilon\right)\right)$, where $\epsilon$ is a sufficiently small positive constant.

Remark 19. With the similar method in Theorem 13 and Theorem 14, we can also discuss the problem of the identification between the unknown parameters $\theta, \psi$ in (7) and $\hat{\theta}$, $\widehat{\psi}$ in $(30)$.

\section{Numerical Simulations}

In this section, we will show that the proposed adaptive controllers are efficient and that the theoretical results are correct. Numerical simulations are performed using MATLAB software. The well-known stochastic chaos between Lorenz system and Chen systems is synchronized using the adaptive controller (15) in the first example. The synchronization between Chen system and Lu system is shown in the second example using the adaptive controller (29). The Lorenz, 


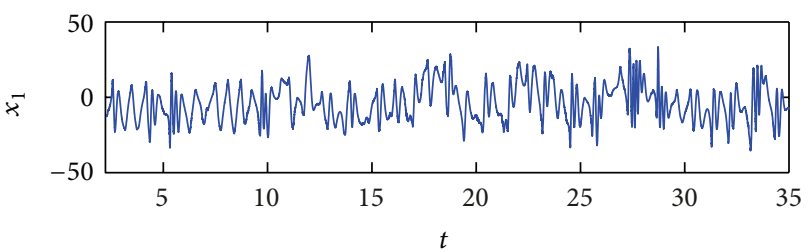

(a)

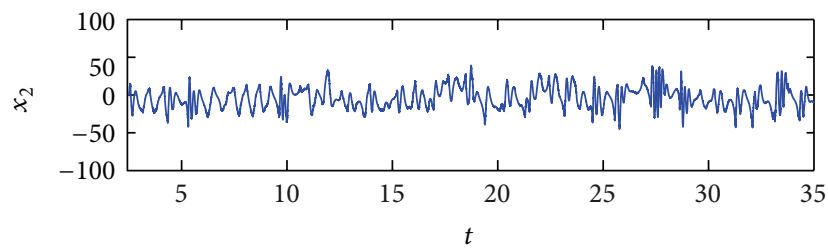

(b)

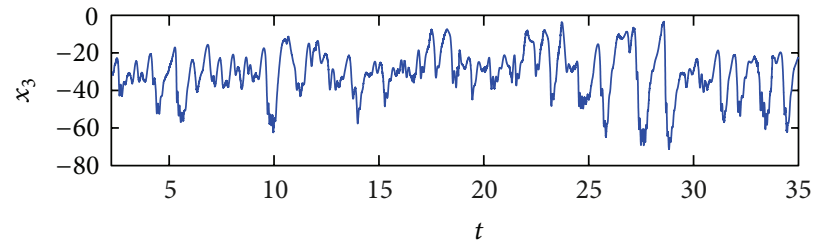

(c)

FIgURE 1: The trajectories of the error system without control input in Example 1.

Chen, and Lu systems are given by the following differential equations, respectively,

$$
\begin{aligned}
& \left\{\begin{array}{l}
\dot{x}_{1}=10\left(x_{2}-x_{1}\right) \\
\dot{x}_{2}=28 x_{1}-x_{2}-x_{1} x_{3} \\
\dot{x}_{3}=x_{1} x_{2}-\frac{8 x_{3}}{3}
\end{array}\right. \\
& \left\{\begin{array}{l}
\dot{x}_{1}=35\left(x_{2}-x_{1}\right) \\
\dot{x}_{2}=28 x_{2}-7 x_{1}-x_{1} x_{3} \\
\dot{x}_{3}=x_{1} x_{2}-3 x_{3}
\end{array}\right. \\
& \left\{\begin{array}{l}
\dot{x}_{1}=36\left(x_{2}-x_{1}\right) \\
\dot{x}_{2}=-20 x_{2}-x_{1} x_{3} \\
\dot{x}_{3}=x_{1} x_{2}-3 x_{3} .
\end{array}\right.
\end{aligned}
$$

In all the cases, the uncertainties $\Delta f(x), \Delta g(y)$, and $\sigma(x)$ and the noise intensity function are given as follows, respectively,

$$
\left\{\begin{array}{l}
\Delta f_{1}(x)=0.5 \sin \left(\pi x_{1}\right), \\
\Delta f_{2}(x)=0.5 \sin \left(2 \pi x_{2}\right), \\
\Delta f_{3}(x)=0.5 \sin \left(3 \pi x_{3}\right),
\end{array}\right.
$$

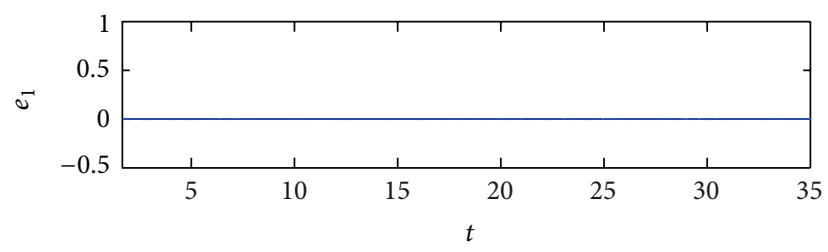

(a)

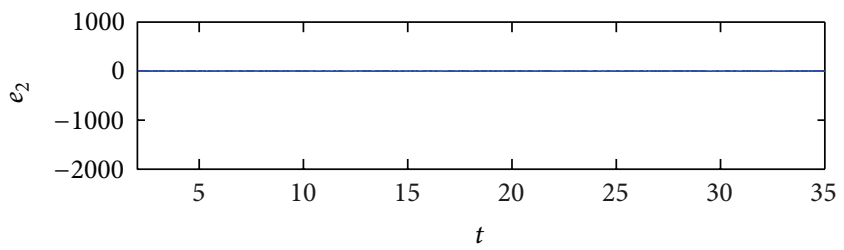

(b)

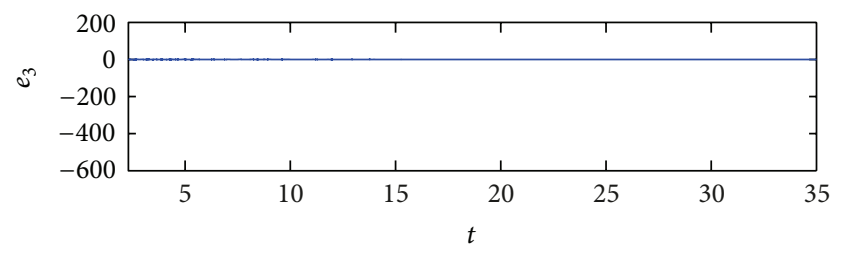

(c)

FIgURE 2: Time responses of error system under control input in Example 1.

$$
\begin{aligned}
& \left\{\begin{array}{l}
\Delta g_{1}(y)=-5 \sin \left(\pi y_{1}\right), \\
\Delta g_{2}(y)=-4 \sin \left(2 \pi y_{2}\right), \\
\Delta g_{3}(y)=-\sin \left(3 \pi y_{3}\right),
\end{array}\right. \\
& \left\{\begin{array}{l}
\sigma_{1}(x)=x_{2}^{2}+0.3 x_{3} \\
\sigma_{2}(x)=x_{1}^{3}+0.1 x_{3} \\
\sigma_{3}(x)=0.2 x_{1}+x_{1} x_{2} .
\end{array}\right.
\end{aligned}
$$

In all simulations, we choose the initial value of the adaptive parameters vectors $\widehat{\theta}_{0}=[5,5,5]^{\mathrm{T}}, \widehat{\psi}_{0}=[3,3,3]^{\mathrm{T}}$, $\widehat{\alpha}_{i 0}=\widehat{\beta}_{i 0}=\widehat{k}_{i j 0}=2$, the constants $k_{1}=10$ and $\epsilon=0.01$.

4.1. Example 1: Synchronization between Lorenz Systems and Chen Systems. The nonlinear part of master and slave systems can be rewritten in the form of (2) and (4) as follows:

$$
\begin{aligned}
& f(x)=\left(\begin{array}{c}
0 \\
-x_{1} x_{3}-x_{2} \\
x_{1} x_{2}
\end{array}\right), \quad F(x)=\left(\begin{array}{ccc}
x_{2}-x_{1} & 0 & 0 \\
0 & x_{1} & 0 \\
0 & 0 & -x_{3}
\end{array}\right) \text {, } \\
& g(y)=\left(\begin{array}{c}
0 \\
-y_{1} y_{3} \\
y_{1} y_{2}
\end{array}\right), \quad G(y)=\left(\begin{array}{ccc}
y_{2}-y_{1} & 0 & 0 \\
-y_{1} & y_{1}+y_{2} & 0 \\
0 & 0 & -y_{3}
\end{array}\right) \text {. }
\end{aligned}
$$




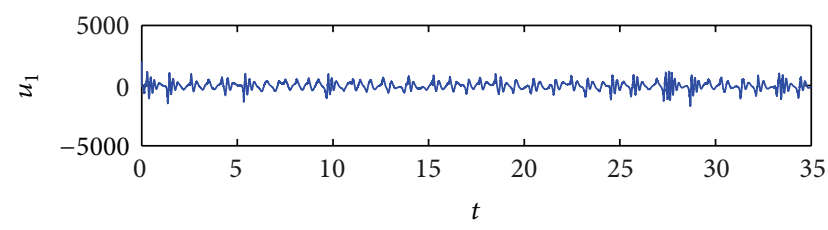

(a)

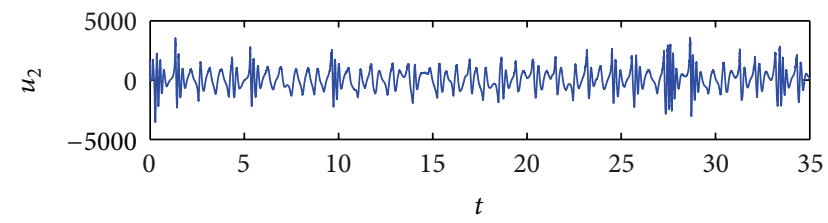

(b)

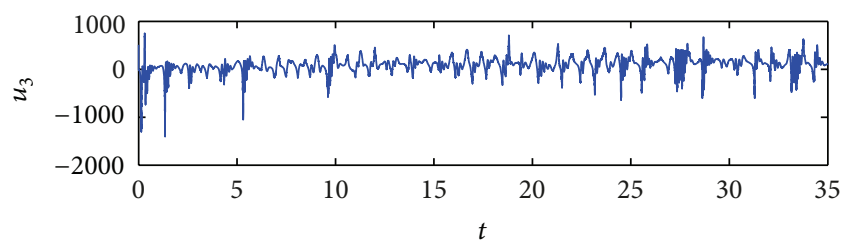

(c)

FIgURE 3: Time response of the control input in Example 1.

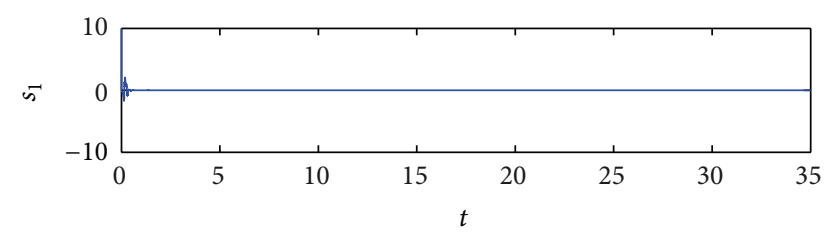

(a)

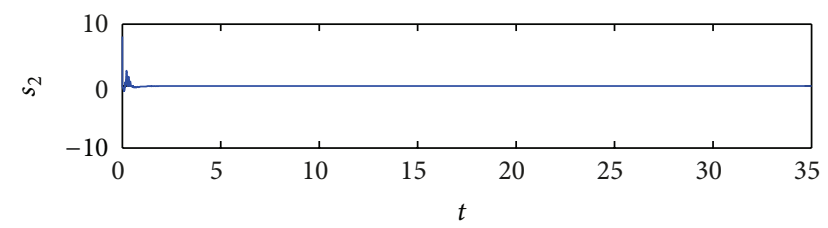

(b)

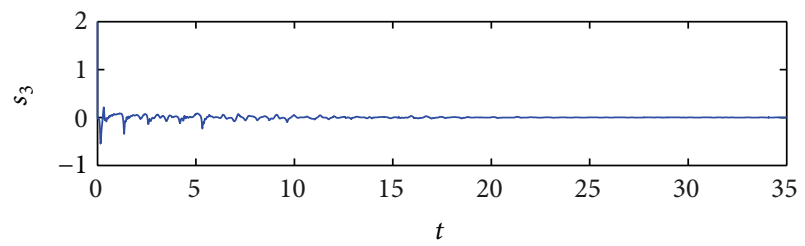

(c)

Figure 4: Time responses of the sliding mode surface $s(t)$ in Example 1.

Consequently, three sliding surfaces are chosen as

$$
\left\{\begin{array}{l}
s_{1}=10 e_{1}(t), \\
s_{2}=8 e_{2}(t), \\
s_{3}=2 e_{3}(t) .
\end{array}\right.
$$

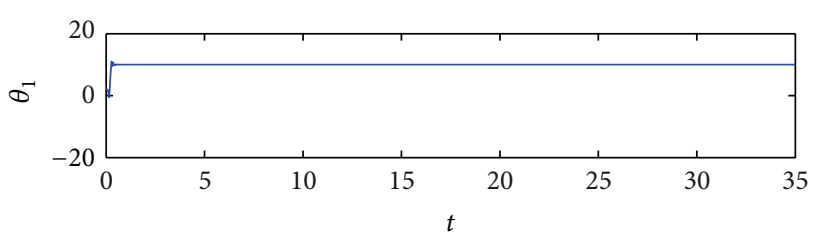

(a)

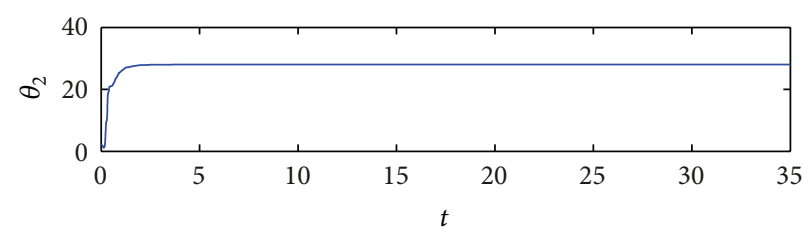

(b)

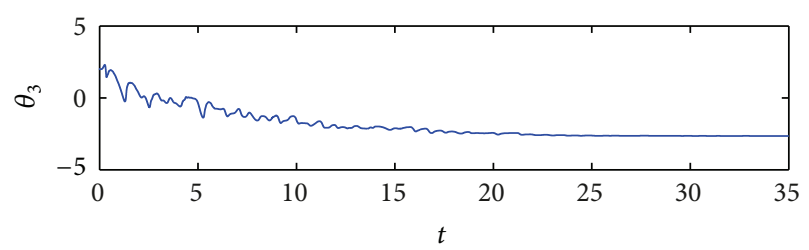

(c)

FIGURE 5: The trajectories of the adaptive laws of the parameter $\widehat{\theta}$ in Example 1.

The stochastic Lorenz and Chen systems are started with the initial conditions as follows: $x_{0}=[1,1.5,2]^{\mathrm{T}}$ and $y_{0}=$ $[2,2.5,3]^{\mathrm{T}}$. The synchronization of the Lorenz and Chen systems without control input is shown in Figure 1 and the error simulation under the control input is shown in Figure 2. As one can see the synchronization errors converge to zero in mean squares. The control input is shown in Figure 3 and the sliding mode surface is shown in Figure 4. The updated vector parameters of $\widehat{\alpha}, \widehat{\beta}, \widehat{\theta}$, and $\widehat{\psi}$ are shown in Figures $5,6,7$, and 8 and $\widehat{k}_{i j}$ are depicted in Figures 9, 10, and 11, respectively. Obviously, all of updated parameters approach some constants.

4.2. Example 2: Synchronization between Chen Systems and Lu Systems. The nonlinear part of master and slave systems can be rewritten in the form of (2) and (4) as follows:

$$
\begin{array}{cc}
f(x)=\left(\begin{array}{c}
0 \\
-x_{1} x_{3} \\
x_{1} x_{2}
\end{array}\right), & F(x)=\left(\begin{array}{ccc}
x_{2}-x_{1} & 0 & 0 \\
-x_{1} & x_{1}+x_{2} & 0 \\
0 & 0 & -x_{3}
\end{array}\right), \\
g(y)=\left(\begin{array}{c}
0 \\
-y_{1} y_{3} \\
y_{1} y_{2}
\end{array}\right), & G(y)=\left(\begin{array}{ccc}
y_{2}-y_{1} & 0 & 0 \\
0 & -y_{2} & 0 \\
0 & 0 & -y_{3}
\end{array}\right) .
\end{array}
$$

Consequently, the same sliding surfaces are chosen as in Example 1. To simplify, we choose the noise intensity function $\sigma(x)=\left[0.4 x_{2}+0.3 x_{3}, 0.2 x_{1}+0.1 x_{3}, 0.3 x_{1}+0.2 x_{2}\right]^{\mathrm{T}}$, so it is easy to see $\alpha_{i}=\beta_{i}=0.5$ and $k_{11}=k_{22}=k_{33}=$ $0, k_{12}=0.4, k_{13}=0.3, k_{21}=0.2, k_{23}=0.1, k_{31}=$ 0.3 , and $k_{32}=0.2$. The stochastic Chen and Lu systems are 


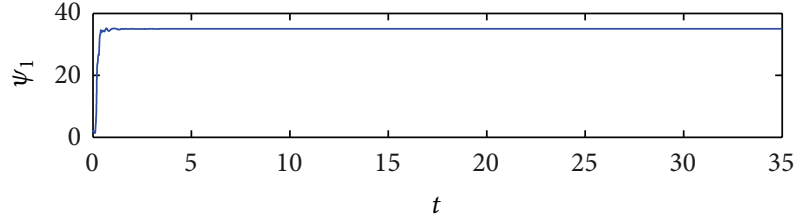

(a)

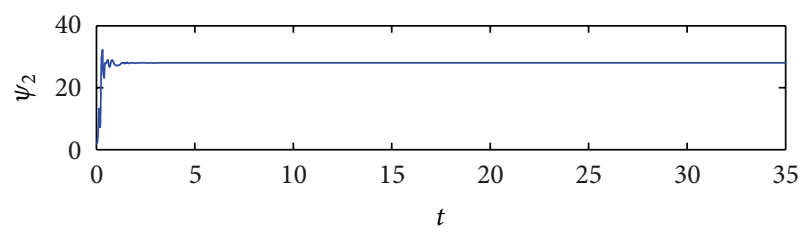

(b)

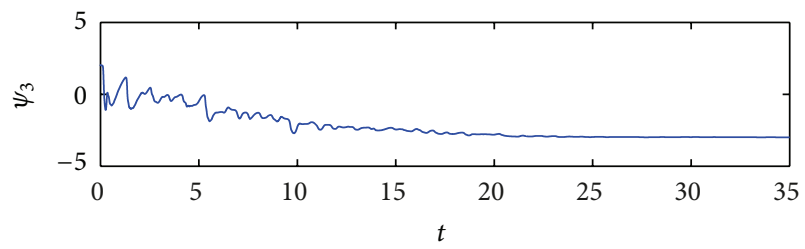

(c)

Figure 6: Time responses of the adaptive update laws $\widehat{\psi}$ in Example 1.

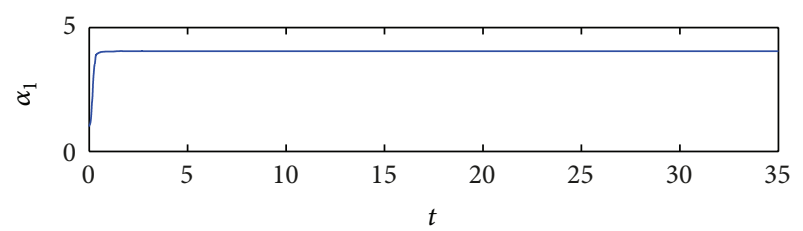

(a)

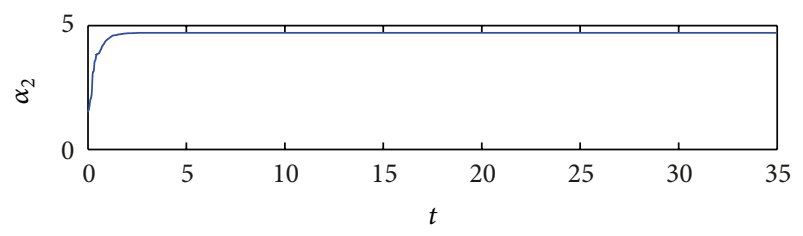

(b)

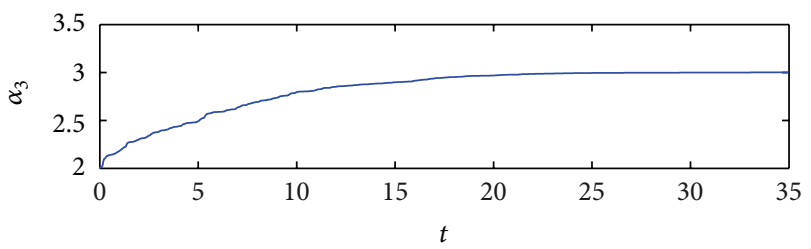

(c)

Figure 7: The trajectories of the adaptive laws $\alpha$ in Example 1.

started with the initial conditions as follows: $x_{0}=[8,4,7]^{\mathrm{T}}$ and $y_{0}=[-10,-4,2]^{\mathrm{T}}$. The synchronization of the Chen and $\mathrm{Lu}$ systems without control input is shown in Figure 12 and the error simulation under the control input is shown in Figure 13. As one can see the synchronization errors converge to zero almost surely. The control input is shown in Figure 14

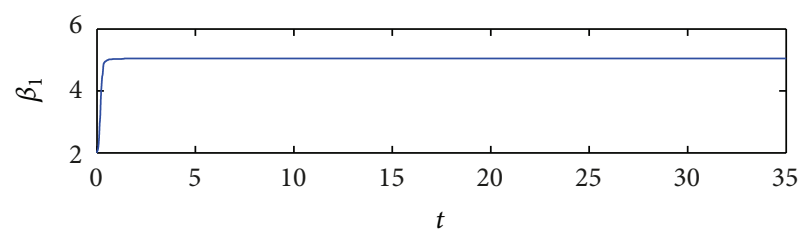

(a)

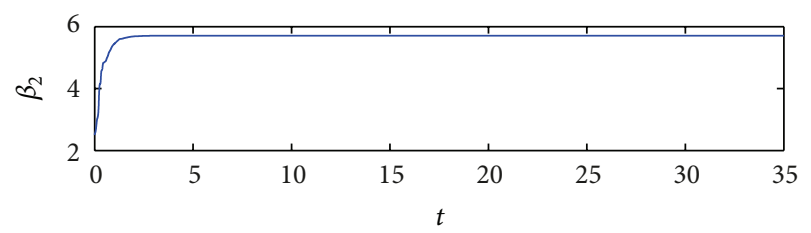

(b)

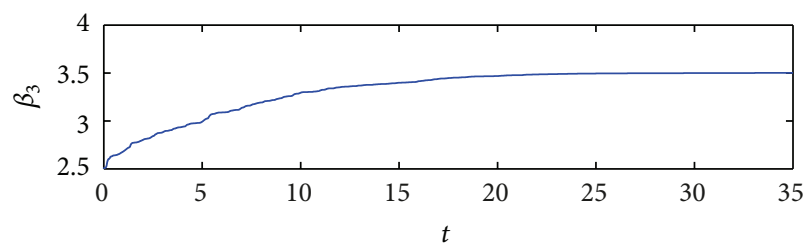

(c)

Figure 8: The trajectories of the adaptive laws $\beta$ in Example 1.

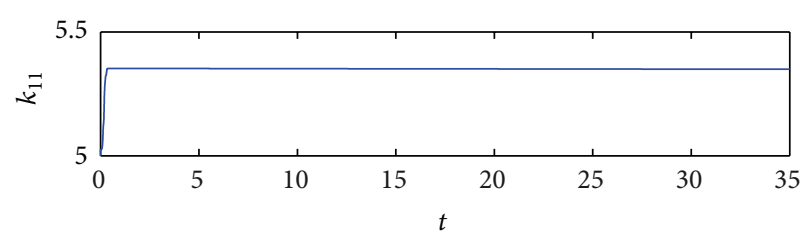

(a)

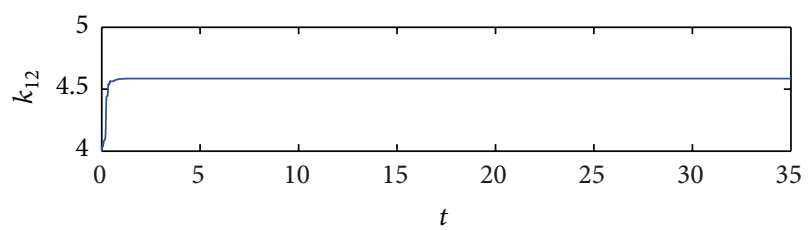

(b)

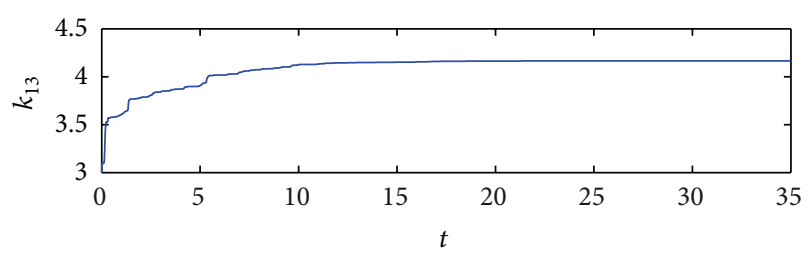

(c)

FIGURE 9: The trajectories of the adaptive laws $k_{11}, k_{12}$, and $k_{13}$ in Example 1.

and the sliding mode surface is shown in Figure 15. The updated vector parameters of $\widehat{\theta}$ and $\widehat{\psi}$ are depicted in Figures 16 and 17, respectively. Obviously, all of updated parameters approach some constants. 


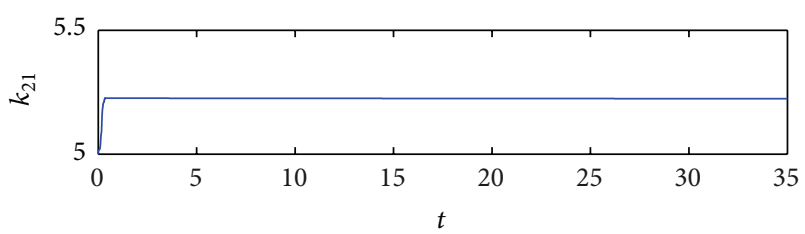

(a)

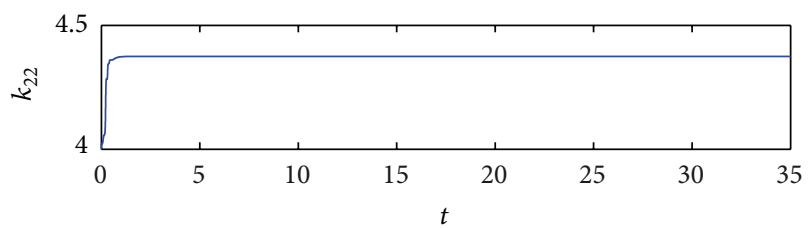

(b)

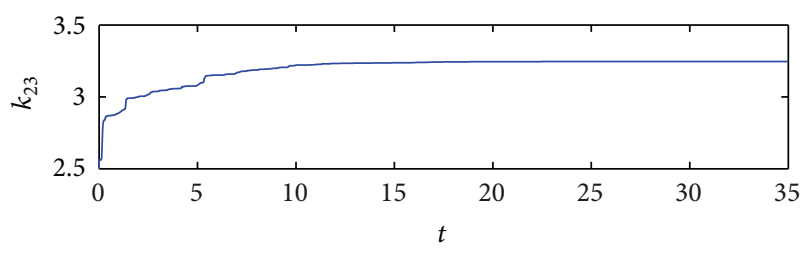

(c)

FIGURE 10: The trajectories of the adaptive laws $k_{21}, k_{22}$, and $k_{23}$ in Example 1.

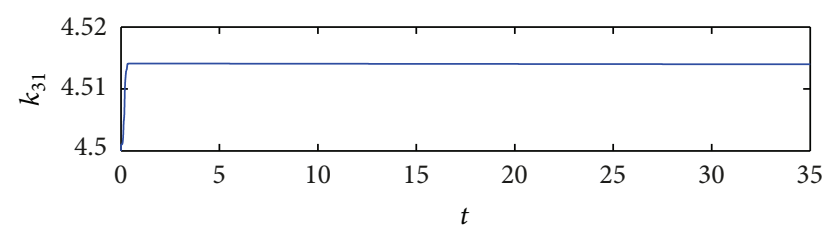

(a)

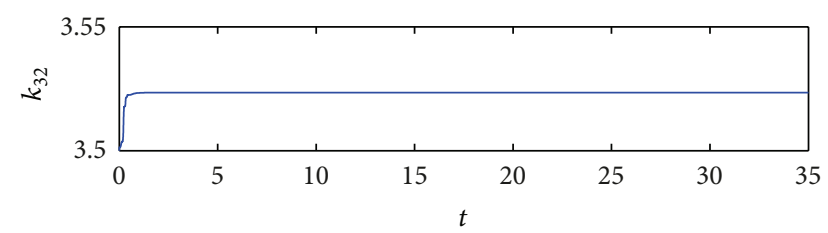

(b)

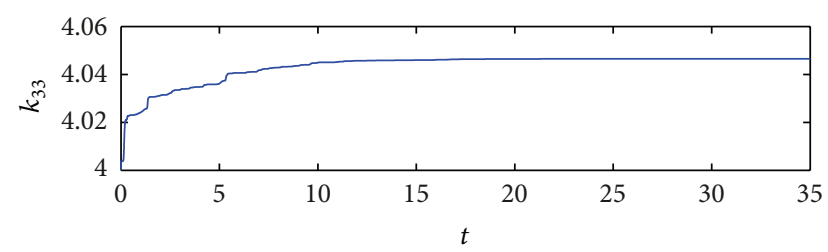

(c)

FIGURE 11: The trajectories of the adaptive laws $k_{31}, k_{32}$, and $k_{33}$ in Example 1.

Remark 20. As it is observed in Figures 5, 6, 16, and 17, the limits of unknown parameter vectors $\widehat{\theta}$ and $\widehat{\psi}$ are not equal to the vectors $\theta$ and $\psi$ in (36). This point is consistent with the results of Theorem 13, Theorem 14, and Remark 19.

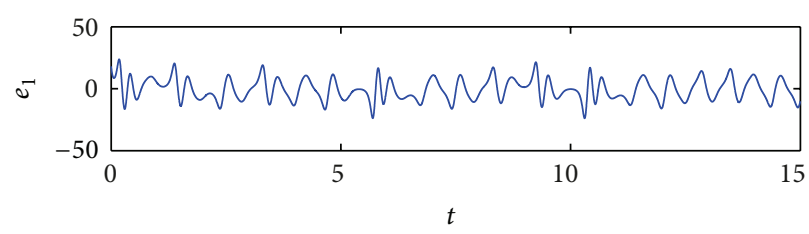

(a)

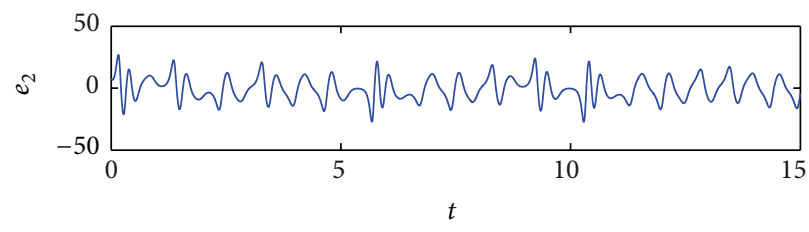

(b)

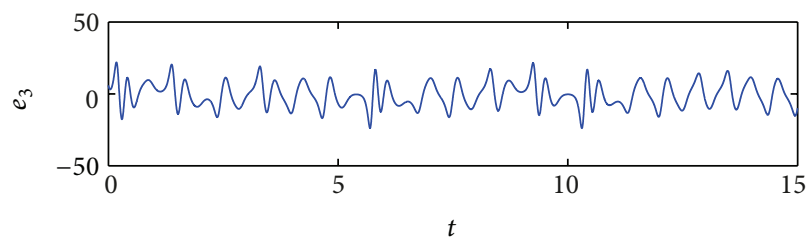

(c)

FIGURE 12: The trajectories of the error system without control input in Example 2.

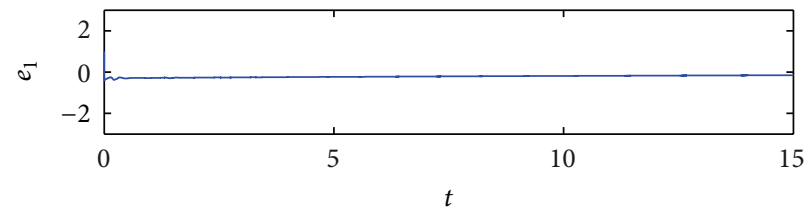

(a)

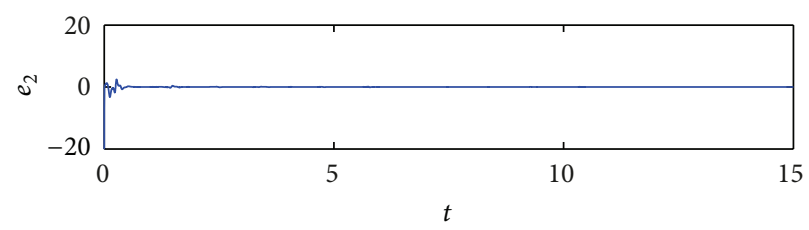

(b)

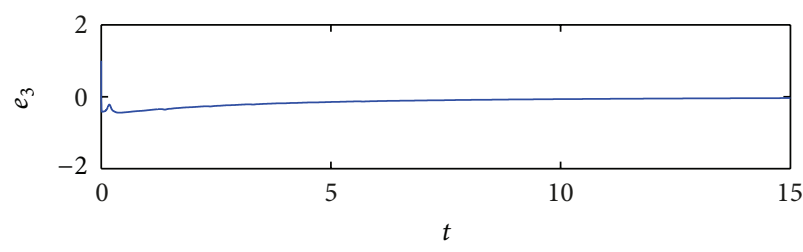

(c)

FIGURE 13: Time responses of error system under control input in Example 2.

\section{Conclusion}

In this paper, adaptive sliding mode controllers are designed to realize the asymptotical synchronization in mean squares and the almost surely synchronization for two different stochastic chaotic systems with unknown parameters and 


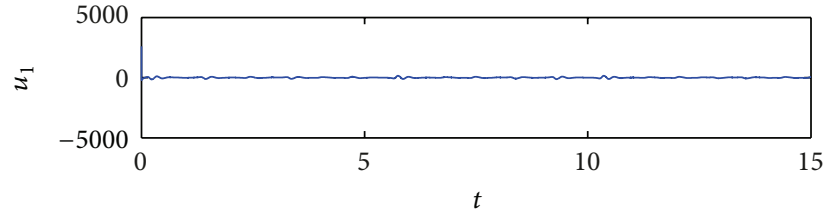

(a)

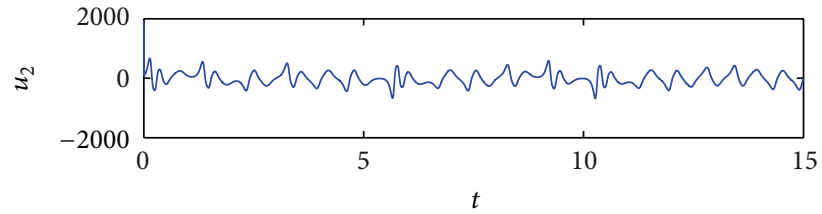

(b)

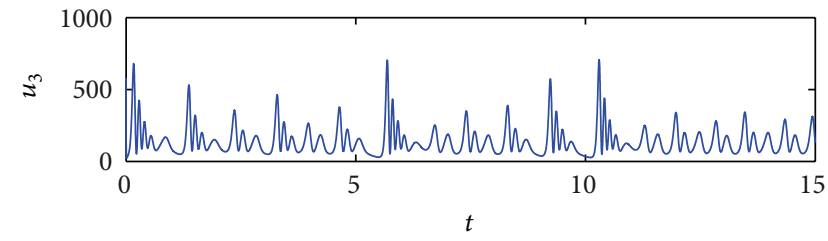

(c)

FIgURE 14: Time responses of the control input in Example 2.

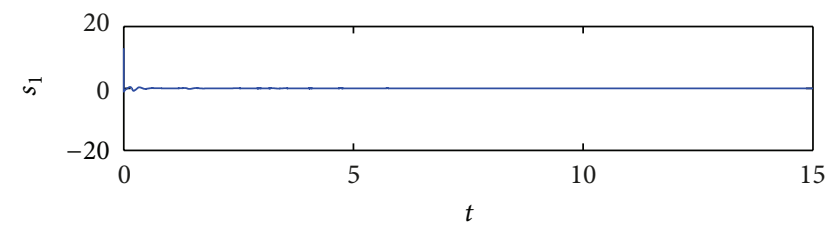

(a)

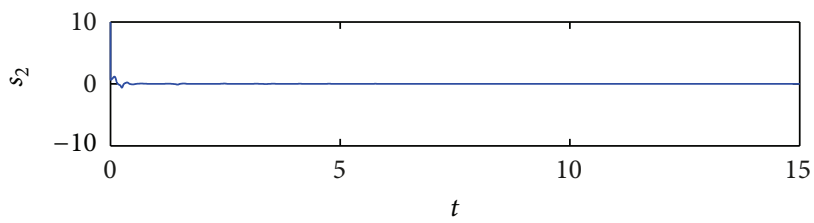

(b)

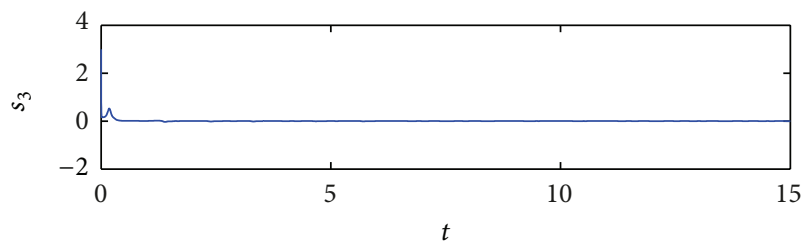

(c)

FIGURE 15: Time responses of the sliding mode surface $s(t)$ in Example 2.

uncertain terms, respectively. The designed controllers' robustness and efficiency are proved between two different pairs of stochastic chaos systems (Lorenz-Chen and ChenLu) with unknown parameters and uncertainties.

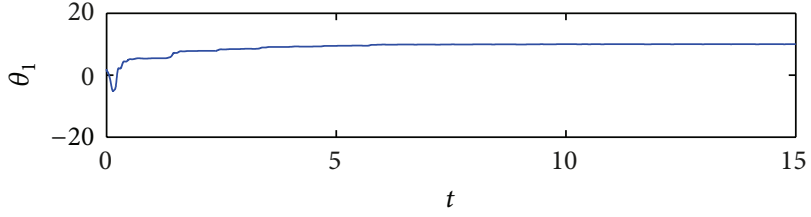

(a)

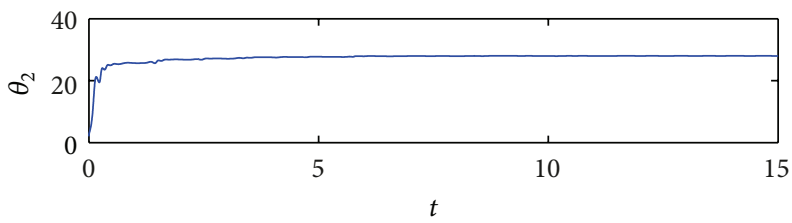

(b)

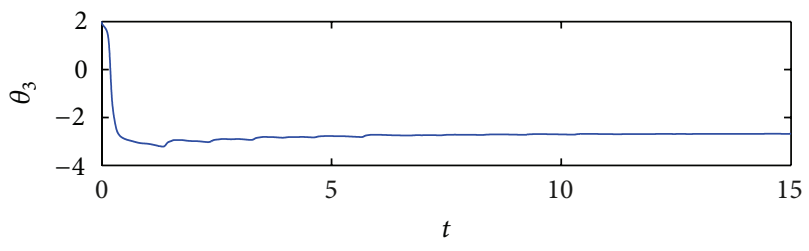

(c)

FIGURE 16: The trajectories of the adaptive laws of the parameter $\hat{\theta}$ in Example 2.

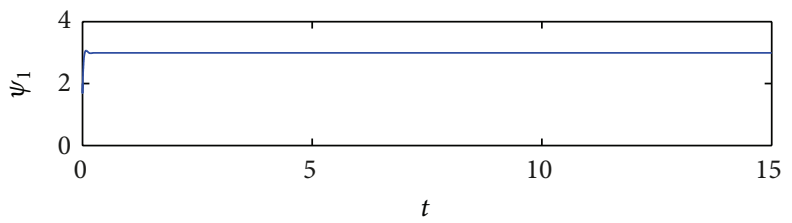

(a)

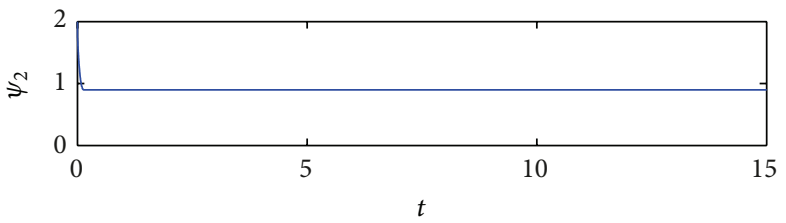

(b)

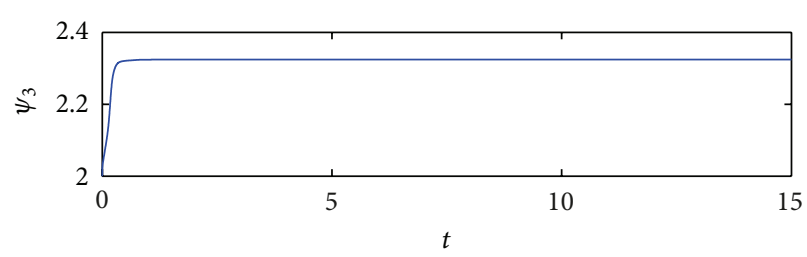

(c)

FIGURE 17: The trajectories of the adaptive laws of the parameter $\widehat{\psi}$ in Example 2.

\section{Acknowledgments}

This work was supported by the National Natural Science Foundation of China (11071060), the Special Funds of the National Natural Science Foundation of China (11226143), the Natural Science Foundation of Hunan Province (13JJ4111), 
the Scientific Research Fund of Hunan Provincial Education Department (11B029), and the Science Foundation of Hunan First Normal University (XYS10N11).

\section{References}

[1] L. M. Pecora and T. L. Carroll, "Synchronization in chaotic systems," Physical Review Letters, vol. 64, no. 8, pp. 821-824, 1990.

[2] T. L. Carroll and L. M. Pecora, "Synchronizing chaotic circuits," IEEE Transactions on Circuits and Systems, vol. 38, no. 4, pp. 453-456, 1991.

[3] L. Kocarev and U. Parlitz, "General approach for chaotic synchronization with applications to communication," Physical Review Letters, vol. 74, no. 25, pp. 5028-5031, 1995.

[4] Y. Yu and S. Zhang, "Adaptive backstepping synchronization of uncertain chaotic system," Chaos, Solitons and Fractals, vol. 21, no. 3, pp. 643-649, 2004.

[5] H. Salarieh and M. Shahrokhi, "Adaptive synchronization of two different chaotic systems with time varying unknown parameters," Chaos, Solitons and Fractals, vol. 37, no. 1, pp. 125136, 2008.

[6] K. Konishi, M. Hirai, and H. Kokame, "Sliding mode control for a class of chaotic systems," Physics Letters, Section A, vol. 245, no. 6, pp. 511-517, 1998.

[7] J. M. Nazzal and A. N. Natsheh, "Chaos control using slidingmode theory," Chaos, Solitons and Fractals, vol. 33, no. 2, pp. 695-702, 2007.

[8] J. Alvarez-Gallegos, "Nonlinear regulation of a Lorenz system by feedback linearization techniques," Dynamics and Control, vol. 4, no. 3, pp. 277-298, 1994.

[9] A. Alasty and H. Salarieh, "Nonlinear feedback control of chaotic pendulum in presence of saturation effect," Chaos, Solitons and Fractals, vol. 31, no. 2, pp. 292-304, 2007.

[10] A. Alasty and H. Salarieh, "Controlling the chaos using fuzzy estimation of OGY and Pyragas controllers," Chaos, Solitons and Fractals, vol. 26, no. 2, pp. 379-392, 2005.

[11] N. Luo and M. de la Sen, "State feedback sliding mode control of a class of uncertain time delay systems," IEE Proceedings D, vol. 140 , no. 4, pp. 261-274, 1993.

[12] N. Luo, M. de la Sen, and J. Rodellar, "Robust stabilization of a class of uncertain time delay systems in sliding mode," International Journal of Robust and Nonlinear Control, vol. 7, no. 1, pp. 59-74, 1997.

[13] M. Pourmahmood, S. Khanmohammadi, and G. Alizadeh, "Synchronization of two different uncertain chaotic systems with unknown parameters using a robust adaptive sliding mode controller," Communications in Nonlinear Science and Numerical Simulation, vol. 16, no. 7, pp. 2853-2868, 2011.

[14] M. P. Aghababa, S. Khanmohammadi, and G. Alizadeh, "Finitetime synchronization of two different chaotic systems with unknown parameters via sliding mode technique," Applied Mathematical Modelling, vol. 35, no. 6, pp. 3080-3091, 2011.

[15] L. Billings, E. M. Bollt, and I. B. Schwartz, "Phase-space transport of stochastic chaos in population dynamics of virus spread," Physical Review Letters, vol. 88, no. 23, pp. 23410112341014, 2002.

[16] W. J. Freeman, "A proposed name for aperiodic brain activity: stochastic chaos," Neural Networks, vol. 13, no. 1, pp. 11-13, 2000.

[17] X. Z. Mou, W. N. Zhou, L. Pan, and Q. Zhu, "Synchronization control of stochastically coupled neural networks with mixed time-delays," in Proceedings of the Chinese Control and Decision Conference (CCDC '09), pp. 3156-3161, June 2009.

[18] Y. Tang, R. Qiu, J.-A. Fang, Q. Miao, and M. Xia, "Adaptive lag synchronization in unknown stochastic chaotic neural networks with discrete and distributed time-varying delays," Physics Letters A, vol. 372, no. 24, pp. 4425-4433, 2008.

[19] X. S. Yang, Q. X. Zhu, and C. X. Huang, "Lag stochastic synchronization of chaotic mixed time-delayed neural networks with uncertain parameters or perturbations," Neurocomputing, vol. 74, no. 10, pp. 1617-1625, 2011.

[20] Z. Y. Wang and L. H. Huang, "Robust decentralized adaptive control for stochastic delayed Hopfield neural networks," Neurocomputing, vol. 74, pp. 3695-3699, 2011.

[21] C. X. Huang, P. Chen, Y. He, L. Huang, and W. Tan, "Almost sure exponential stability of delayed Hopfield neural networks," Applied Mathematics Letters, vol. 21, no. 7, pp. 701-705, 2008.

[22] C. X. Huang and J. D. Cao, "Almost sure exponential stability of stochastic cellular neural networks with unbounded distributed delays," Neurocomputing, vol. 72, no. 13-15, pp. 3352-3356, 2009.

[23] Y. Z. Sun and J. Ruan, "Synchronization between two different chaotic systems with noise perturbation," Chinese Physics Letters, vol. 19, no. 7, Article ID 070513, 2010.

[24] H. Salarieh and A. Alasty, "Adaptive chaos synchronization in Chua's systems with noisy parameters," Mathematics and Computers in Simulation, vol. 79, no. 3, pp. 233-241, 2008.

[25] H. Salarieh and A. Alasty, "Control of stochastic chaos using sliding mode method," Journal of Computational and Applied Mathematics, vol. 225, no. 1, pp. 135-145, 2009.

[26] X. Mao, Stochastic Differential Equation and Application, Horwood, Chichester, UK, 1997.

[27] R. Z. Khasminskii, Stochastic Stability of Differential Equations, vol. 7 of Monographs and Textbooks on Mechanics of Solids and Fluids: Mechanics and Analysis, Sijthoff \& Noordhoff, Amsterdam, The Netherlands, 1980.

[28] X. Mao, "A note on the LaSalle-type theorems for stochastic differential delay equations," Journal of Mathematical Analysis and Applications, vol. 268, no. 1, pp. 125-142, 2002.

[29] Y. Shen, Q. Luo, and X. Mao, "The improved LaSalle-type theorems for stochastic functional differential equations," Journal of Mathematical Analysis and Applications, vol. 318, no. 1, pp. 134154, 2006.

[30] M. Chen and W. H. Chen, "Robust adaptive neural network synchronization controller design for a class of time delay uncertain chaotic systems," Chaos, Solitons and Fractals, vol. 41, no. 5, pp. 2716-2724, 2009. 


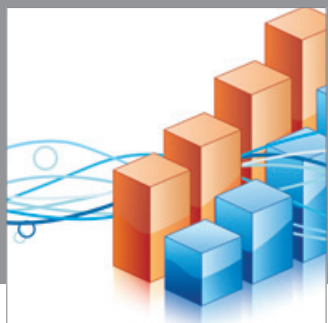

Advances in

Operations Research

mansans

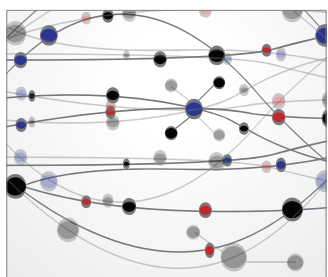

The Scientific World Journal
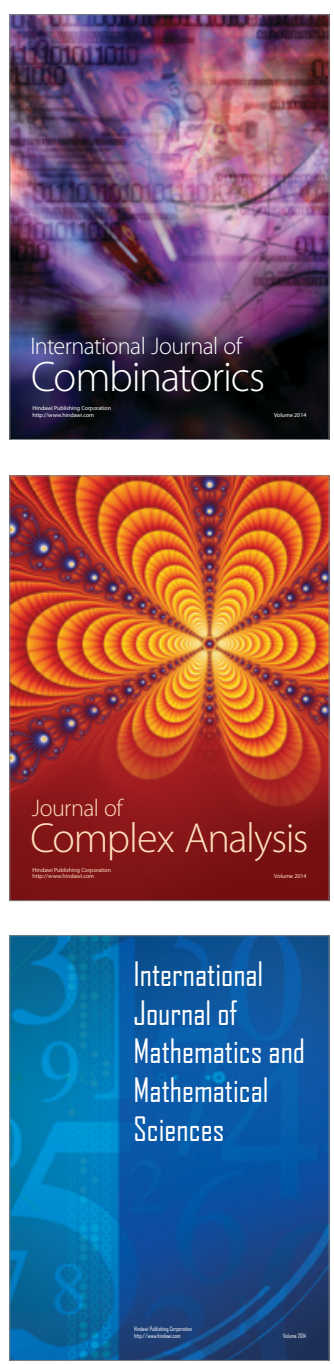
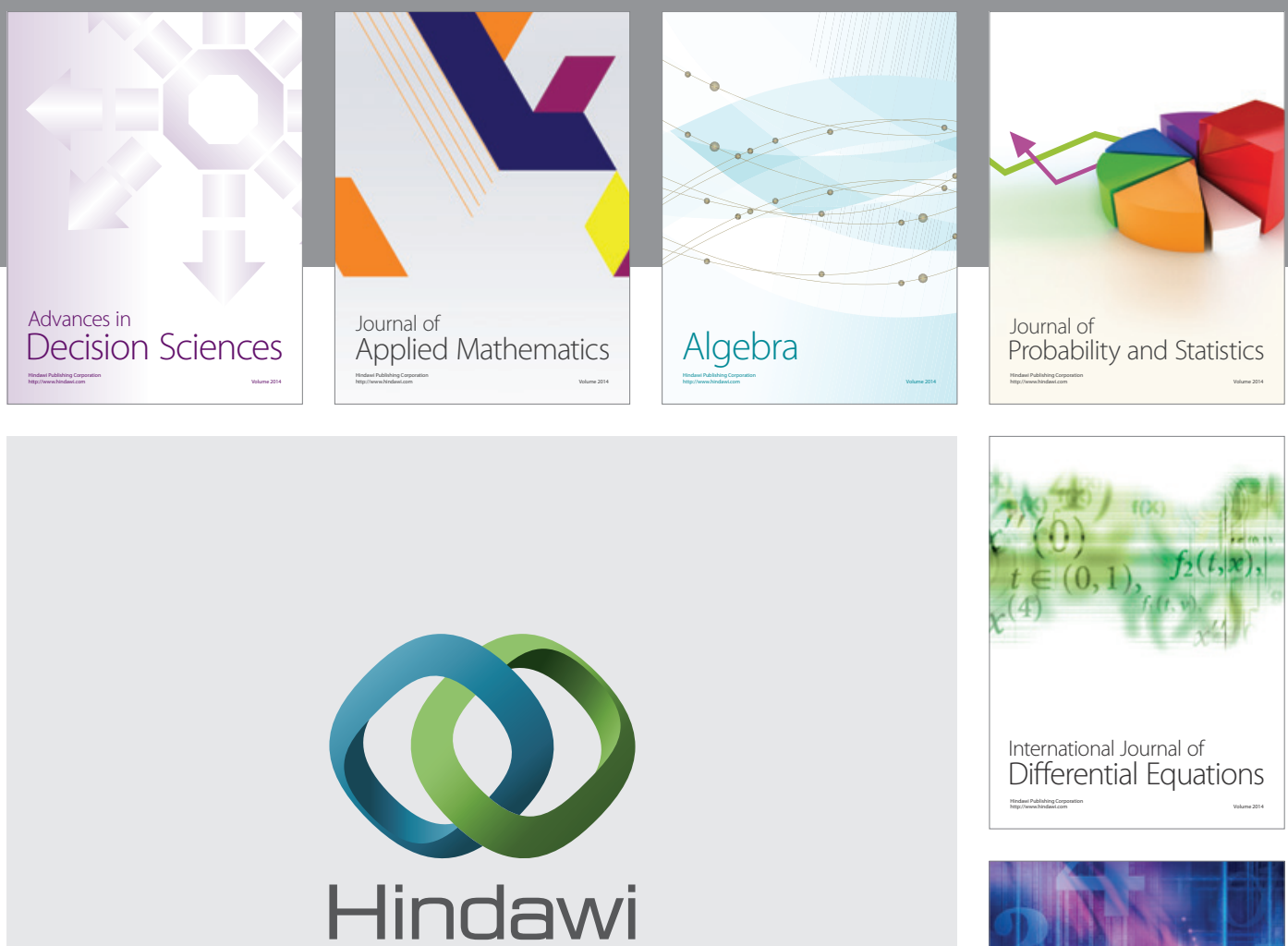

Submit your manuscripts at http://www.hindawi.com
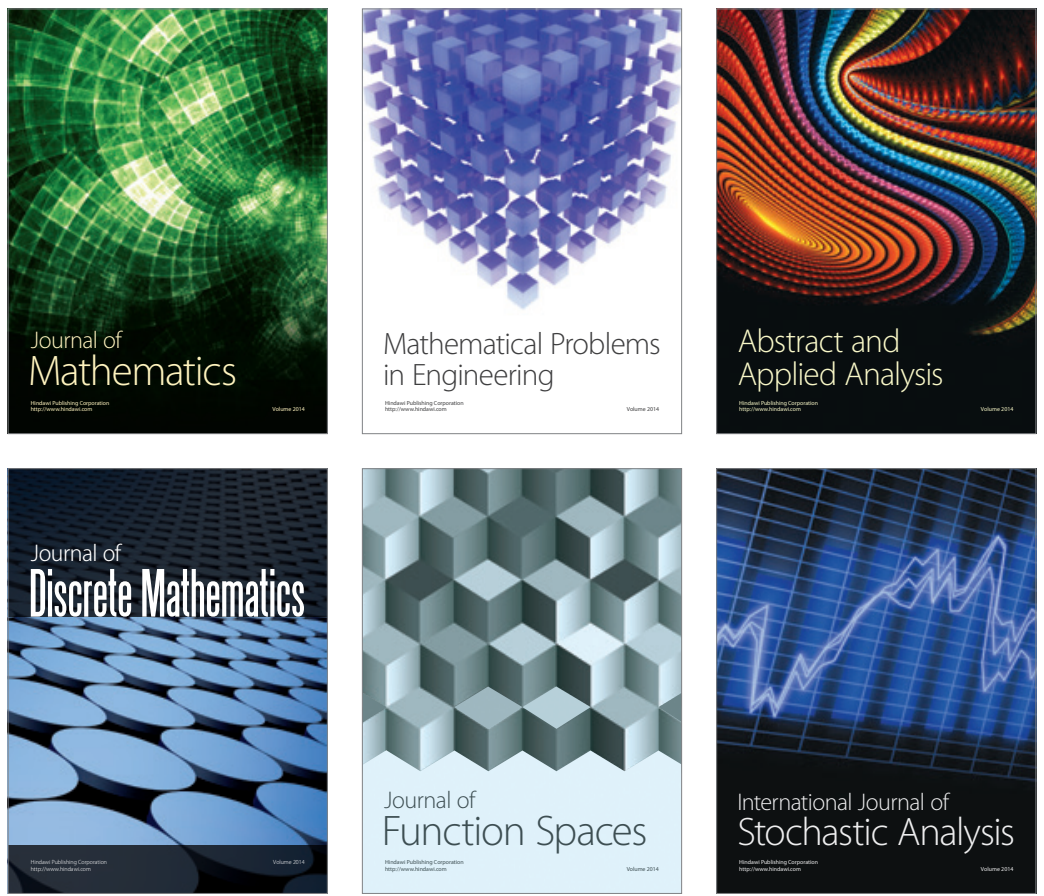

Journal of

Function Spaces

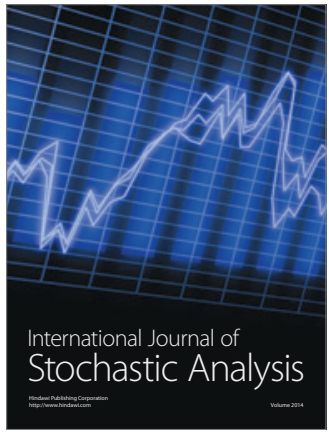

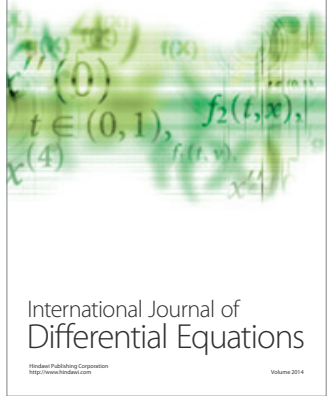
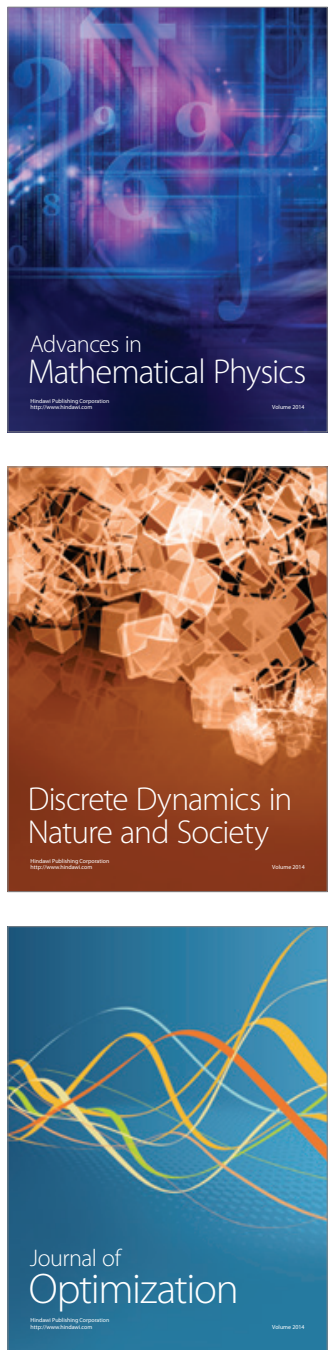